\title{
Topical, geospatial, and temporal diffusion of the 2015 North American Menopause Society position statement on nonhormonal management of vasomotor symptoms
}

\author{
Janet S. Carpenter, PhD, RN, FAAN, ${ }^{1}$ Tei Laine, PhD, ${ }^{2}$ Blake Harrison, $M S,{ }^{2}$ Meghan LePage, BA, ${ }^{2}$ \\ Taran Pierce, MSPH, ${ }^{3}$ Nathan Hoteling, PhD, ${ }^{2}$ and Katy Börner, $P h D^{2,4}$
}

\begin{abstract}
Objective: We sought to depict the topical, geospatial, and temporal diffusion of the 2015 North American Menopause Society position statement on the nonhormonal management of menopause-associated vasomotor symptoms released on September 21, 2015, and its associated press release from September 23, 2015.

Methods: Three data sources were used: online news articles, National Public Radio, and Twitter. For topical diffusion, we compared keywords and their frequencies among the position statement, press release, and online news articles. We also created a network figure depicting relationships across key content categories or nodes. For geospatial diffusion within the United States, we compared locations of the 109 National Public Radio (NPR) stations covering the statement to 775 NPR stations not covering the statement. For temporal diffusion, we normalized and segmented Twitter data into periods before and after the press release (September 12, 2015 to September 22, 2015 vs September 23,2015 to October 3,2015 ) and conducted a burst analysis to identify changes in tweets from before to after.

Results: Topical information diffused across sources was similar with the exception of the more scientific terms "vasomotor symptoms" or "vms" versus the more colloquial term "hot flashes." Online news articles indicated media coverage of the statement was mainly concentrated in the United States. NPR station data showed similar proportions of stations airing the story across the four census regions (Northeast, Midwest, south, west; $P=0.649$ ). Release of the statement coincided with bursts in the menopause conversation on Twitter.
\end{abstract}

Conclusions: The findings of this study may be useful for directing the development and dissemination of future North American Menopause Society position statements and/or press releases.

Key Words: Guidelines - Hot flashes - Information science - Innovation diffusion - Menopause.

I n fall of 2015, The North American Menopause Society (NAMS) published a position statement on the nonhormonal management of menopause-associated vasomotor

Received November 14, 2016; revised and accepted February 14, 2017. From the ${ }^{1}$ Department of Science of Nursing Care, School of Nursing, Indiana University, Indianapolis, IN; ${ }^{2}$ Department of Information and Library Science, School of Informatics and Computing, Indiana University, Bloomington, IN; ${ }^{3}$ Division of Global Health Protection, Center for Global Health, Centers for Disease Control and Prevention, Atlanta, GA; and ${ }^{4}$ Indiana University Network Science Institute, Indiana University, Bloomington, IN.

Disclaimer: The findings and conclusions in this report are those of the authors and do not necessarily represent the official position of the Center for Disease Control and Prevention or the official views of the National Institutes of Health.

Funding/support: This work was partially funded by the National Institutes of Health under award U01CA198934 and P01AG039347 and made possible through student and faculty participation in the spring 2016 Indiana University Information Visualization Massive Open Online Course.

Financial disclosure/conflicts of interest: None reported.

Supplemental digital content is available for this article. Direct URL citations appear in the printed text and are provided in the HTML and PDF versions of this article on the journal's Website (www.menopause.org). Address correspondence and reprints to: Janet S. Carpenter, PhD, RN, FAAN, Distinguished Professor, Indiana University School of Nursing, 600 Barnhill Drive, Indianapolis, IN 46202. E-mail: carpentj@iu.edu symptoms. ${ }^{1}$ The statement was developed by a panel of experts who reviewed the literature and made evidence-based recommendations for clinicians and the public about whether and what nonhormonal therapies were recommended. The paper was publicly available on September 21, 2015 and a press release issued by NAMS on September 23, 2015 appeared widely in the media.

Although NAMS regularly issues position statements, to our knowledge, there has been no analysis of their topical, geospatial, or temporal diffusion. According to the Diffusion of Innovations theory, the spread of knowledge is foundational for deciding to accept and implement change. ${ }^{2}$ Thus, understanding how knowledge of the position statement and its content was spread or diffused by the media could provide foundational information for understanding the position statement's reach and impact. For example, it is important to ascertain how the content was portrayed in different media, which news outlets posted/published it, whether any news outlets acted as super spreaders of information, and the length of time the information was in the public's eye. Such information can be used by NAMS, media outlets, clinicians, and consumers to ensure that knowledge contained within a position 


\section{CARPENTER ET AL}

statement is being released and understood in the most timely and accurate manner possible.

The purpose of this study was to evaluate the temporal, geospatial, and topical diffusion of the aforementioned 2015 NAMS position statement. ${ }^{1}$ We sought to answer the following research questions:

1. Topical: Did different facts spread through different pathways?

2. Geospatial: In what geographic locations was the position statement covered by the media?

3. Temporal: Were there bursts of activity in social media conversations (ie, Twitter) after the statement and press release appeared? What was the length of time the information was highlighted in the media?

\section{METHODS}

\section{Data selection}

There were three sources of data: online news articles; US National Public Radio (NPR); and Twitter. The primary dataset consisted of a collection of online news articles covering the NAMS position statement and press release. We documented press coverage in real time using Google and the keywords "North American Menopause Society" or "NAMS." We used the "Explore in Depth" feature to choose articles grouped by Google based on similarity. At the time of the analysis (spring 2016), we had 24 articles with working URLs from news sites and lifestyle/health blogs mostly within North America (United States, Canada) and from Australia, the Isle of Mann, Malaysia, and the United Kingdom (see Supplemental Table, http://links.lww.com/ MENO/A229). The second and supporting dataset included geographic information pertaining to NPR stations across the United States that covered $(n=109)$ or did not cover $(n=775)$ the position statement and/or press release. The third dataset was a collection of social media data from Twitter for the 11-day periods before (September 12, 2015 to September 22, 2015) and after (September 23, 2015 to October 3, 2015) the publication of the position statement created using a query with keywords related to the NAMS statement. In all, 12,642 tweets were captured through a caseinsensitive query, pairing the following key terms and phrases together: "North American Menopause Society," "NAMS,', " "menopause," " "nonhormonal therapies," " "hot flashes,", and " "vasomotor symptoms.", This query was constructed specifically to collect tweets that could potentially correlate with the public release of the NAMS statement.

\section{Data processing}

For the online news articles, the text content of the web pages from the 24 working URLs from news sites and lifestyle/health blogs was fetched and all irrelevant and redundant content was removed (eg, publication details, author information, running footers and headers, and menu lists), retaining only the title, subtitles, and the body of the web pages. The URLs are listed in the Supplemental Table (http://links.lww.com/MENO/A229). To create the final dataset, these were combined with the name of the news site or lifestyle/health blog, the geographical location of the publication source (if known), the original URL, and the publication date (year, month, day). For the NPR data collected from the 109 NPR stations, we included the syndicated article once, rather than repeating that content 109 times. We then created a table with the 10 most frequent words from the position statement, press release, and online news articles. The numbers in the table and the total word counts, and also the number of unique words, reflected totals (not means) over all the articles, counting the NPR release only once. These counts included words in the title, subtitles, and article body only (not author or publication information, or footers and headers). The counts were derived from a tool used for creating some word clouds for the working analysis (not shown in this paper). The counts do not include stop words, such as articles or prepositions, but count the variations of the same word as separate entries (eg, studies, studying, studied).

To complete the network of topical diffusion, we manually identified and coded statements into one of six distinct categories: treatments recommended; treatments recommended with caution; treatments not recommended; statistical references; quotations from the expert panel chair; and independent expert opinions. In general, the content of each fell completely within one of these categories, and in most cases the statements were consistent among articles. The network was laid out using Gephi ${ }^{3}$ so that nodes were sized according to the number of individual items contained within the article, and each individual item was sized according to the number of articles.

To help identify any potential trends or patterns in geospatial diffusion of the NAMS message, we used the latitude and longitude GPS coordinates available for all the US NPR stations to generate a map distinguishing which stations did and did not play the NAMS story. ${ }^{4,5}$ We calculated the number of stations that did and did not air the story in each region and compared them using chi-square to evaluate whether coverage was greater in one geographic region over another (https://www.fcc.gov/media/radio/fm-query).

For temporal analysis, we analyzed dates the online news articles appeared using frequency counts of Twitter data. We segmented the normalized Twitter data into two periodsbefore the press release (September 12, 2015 to September 22, 2015) and after (September 23, 2015 to October 3, 2015). ${ }^{4}$ There was an average of 575 tweets per day during the time frame; the day with the most tweets was September 29, 2015, with 738 , and the day with the fewest tweets was September 19, 2015, with 414. We generated a selection of keywords, including the top general keywords used in the original filtering of the Twitter data, and all treatments mentioned in the statement. Using the stemmed version of the keywords, we generated word counts for each of the words in the before and after period. Words that returned no hits were excluded. Then, a double-sided bar chart was generated to display the frequency of each keyword. 
NAMS PRESS COVERAGE

TABLE 1. Top 10 most frequently diffused words by source and commonality ${ }^{a}$

\begin{tabular}{|c|c|c|c|c|c|}
\hline \multicolumn{2}{|c|}{ NAMS position statement } & \multicolumn{2}{|c|}{ NAMS press release } & \multicolumn{2}{|c|}{ Online news articles } \\
\hline \multicolumn{6}{|c|}{ Common to all three } \\
\hline Women & 85 & Women & 10 & Women & 145 \\
\hline Evidence & 74 & Evidence & 5 & Evidence & 78 \\
\hline Hot & 51 & Hot & 10 & Hot & 197 \\
\hline Therapies & 37 & Therapies & 9 & Therapies, therapy & 107,77 \\
\hline \multicolumn{6}{|c|}{ Common to press release and news articles } \\
\hline & & Flashes & 7 & Flashes & 162 \\
\hline & & Panel & 9 & Panel & 107 \\
\hline & & Work & 5 & Work & 78 \\
\hline \multicolumn{6}{|c|}{ Unique words by source } \\
\hline VMS & 110 & Approaches & 7 & Effective & 67 \\
\hline Level & 66 & Providers & 6 & NAMS & 66 \\
\hline Trials & 39 & Can & 5 & & \\
\hline Studies & 39 & & & & \\
\hline Randomized & 38 & & & & \\
\hline Group & 35 & & & & \\
\hline
\end{tabular}

NAMS, The North American Menopause Society; NPR, National Public Radio; VMS, vasomotor symptoms.

${ }^{a}$ Words in bold represent those unique to the source. The numbers reflect totals (not means) over all the articles, counting the NPR release only once.

These counts included words in the title, subtitles, and article body only (not author or publication information, or footers and headers). The counts do not include stop words, such as articles or prepositions, but count the variations of the same word as separate entries (eg, studies, studying, studied).

\section{RESULTS}

\section{Topical diffusion}

Table 1 shows the most frequent words in the position statement, press release, and press coverage. Total word counts for each source were 10,568, 715, and 13,434 words, respectively. Unique words in each source were 2,421, 292, and 1,508 , respectively.

Figure 1 depicts the network of topical diffusion. Topical content nodes are placed along the left half of the circle; NAMS publications and media outlets are depicted on the right. In general, each individual article included some key items within each of the three recommendation categories. All articles showed significant overlap with the press release; few included text from the position statement that was not included in the press release. Some articles referenced comments from outside experts, in particular Today and Tech Times, and each included quotes from three different experts. Others were weighted more heavily on numbers and statistics from the press release and the position statement, or on quotations from the expert panel chair that were contained in the press release.

\section{Geospatial diffusion}

Table 2 shows the number of NPR stations that did and did not air the story within each of the four US Census regions. Although the majority of NPR stations did not run the NAMS position statement story, those that did were widely dispersed across the United States. There was no significant difference in NPR coverage across the four regions which was an indication that the NAMS position statement was covered throughout the United States and had a wide reach and audience. The supplemental figure (http://links.lww.com/MENO/A230) shows the NPR station coverage across the United States.

\section{Temporal diffusion}

Among the articles with available publishing dates, most of the coverage spanned less than 2 weeks from September
23, 2015 to October 5, 2015 after the press release, and only as few as 4 articles were published from 2 to 5 months after the initial press release. By analyzing the unique articles (ie, counting the syndicated NPR article once), 58\% were published on September 23, 2015 and a cumulative 79\% were published on September 23, 2015 or September 24, 2015.

Figure 2 shows results of the Twitter data analysis. For most of the keywords searched, the frequency of their appearance in tweets showed a statistically significant increase in the period after the press release compared with the period before the release. We removed 7 keywords with the lowest frequencies both before and after, and conducted a chi-square test of differences, which indicated more tweets in the period after the press release compared with the period before $\left(\chi^{2}[18, \mathrm{~N}=13,193]=165.6 ; P<0.001\right)$.

\section{DISCUSSION}

Our analysis of content diffusion revealed that, in general, the coverage details across media outlets were similar, without significant variation in topics covered, and largely reflective of the press release. Each story identified a handful of treatments across the three categories: recommended, recommended with caution, and not recommended. The stories did, however, show some variation in how supporting information was used, such as the use of outside experts, statistical references, and quotations from the chair of the expert panel. Interestingly, most of the media coverage comprised statements, quotations, and facts that were directly available from the press release, with a few articles showing evidence that information had been obtained from the original article. While this finding is intriguing within the context of this study, more data from similar studies are needed before any broad statements or conclusions can be formulated. Nevertheless, this finding does point to the importance of an accurate and carefully crafted press release. 


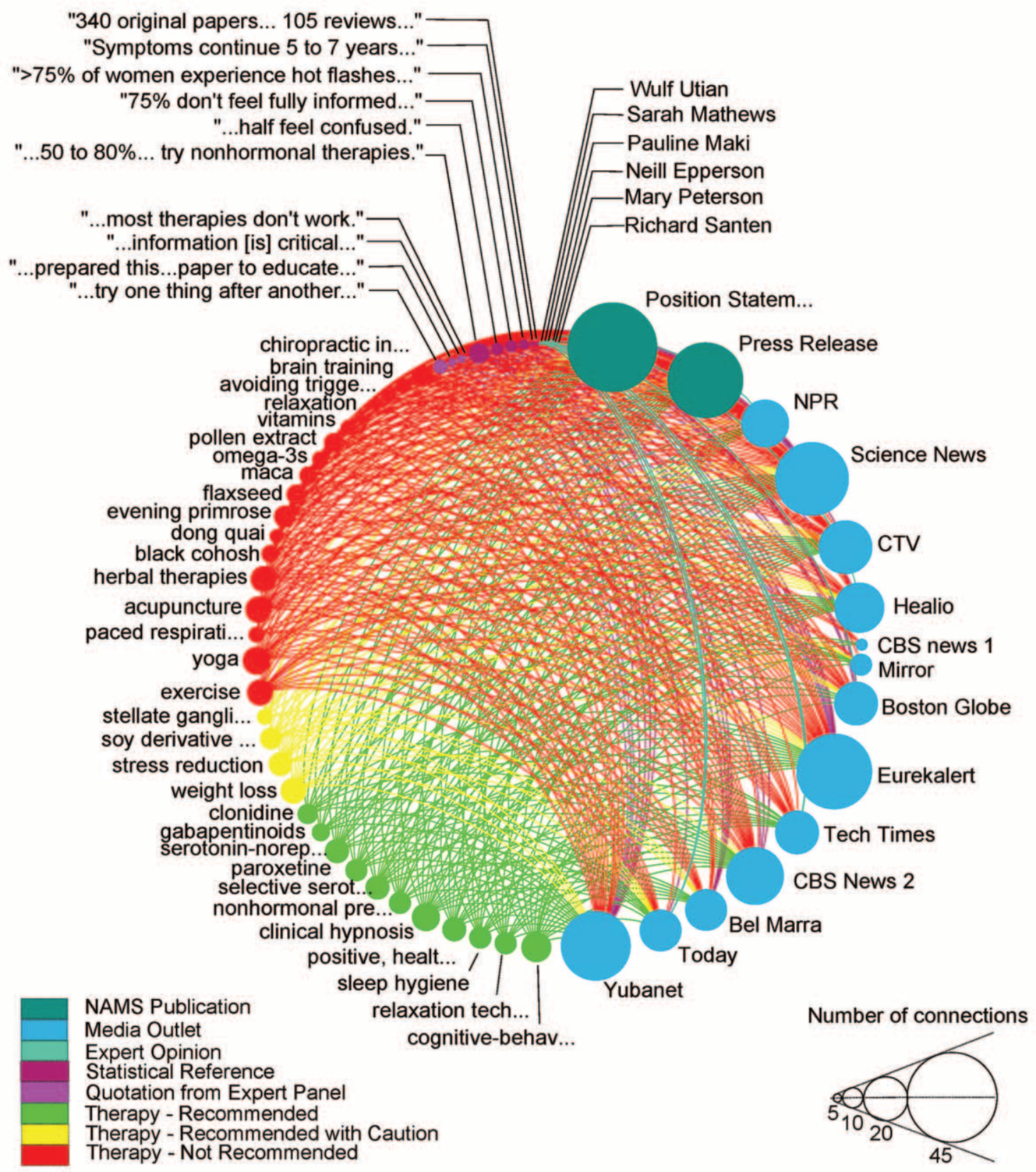

FIG. 1. Topical diffusion network of the North American Menopause Society (NAMS) Position Statement in the Media. Topical analysis of the media coverage of the NAMS position statement is shown here. On the right hand side of the circular layout are the two NAMS publications (teal circles) and publications by different Media Outlets (blue circles). On the left hand side are recommended therapies (green), therapies recommended with caution (yellow), not recommended therapies (red), and also quotation from expert panels (purple), statistical references (dark red), and single expert opinions (turquoise). Nodes are sized according to the number of topical items that were included in the article or the number of media outlets, which included that item. Edges connect NAMS publications and Media Outlet nodes on the right to topical nodes on the left. Edge colors are assigned based on the topical content that was linked to a given NAMS publication or media outlet. CBS, Columbia Broadcasting System; CTV, Canadian Television; NPR, National Public Radio.

Our analysis of geospatial diffusion shows broad coverage across the United States for online news articles and NPR stories. NPR station data were not more concentrated within one census region over another. Future studies should also identify the time of day that the story was aired at each station, to obtain a correlation with the expected audience demographics. Nearly all online news sources were from North America (United States and Canada), and only a few articles
TABLE 2. Geospatial diffusion of NPR coverage of the NAMS position statement across US Census regions

\begin{tabular}{lccccc}
\hline & Northeast & Midwest & South & West & Total \\
\hline Aired NAMS & 20 & 29 & 36 & 24 & 109 \\
Did not air & 179 & 211 & 240 & 145 & 775 \\
Total & 199 & 240 & 276 & 169 & 884 \\
\hline
\end{tabular}

$\chi^{2}=1.648_{(\mathrm{df}=3)}, P=0.649$.

NAMS, The North American Menopause Society; NPR, National Public Radio. 
NAMS PRESS COVERAGE

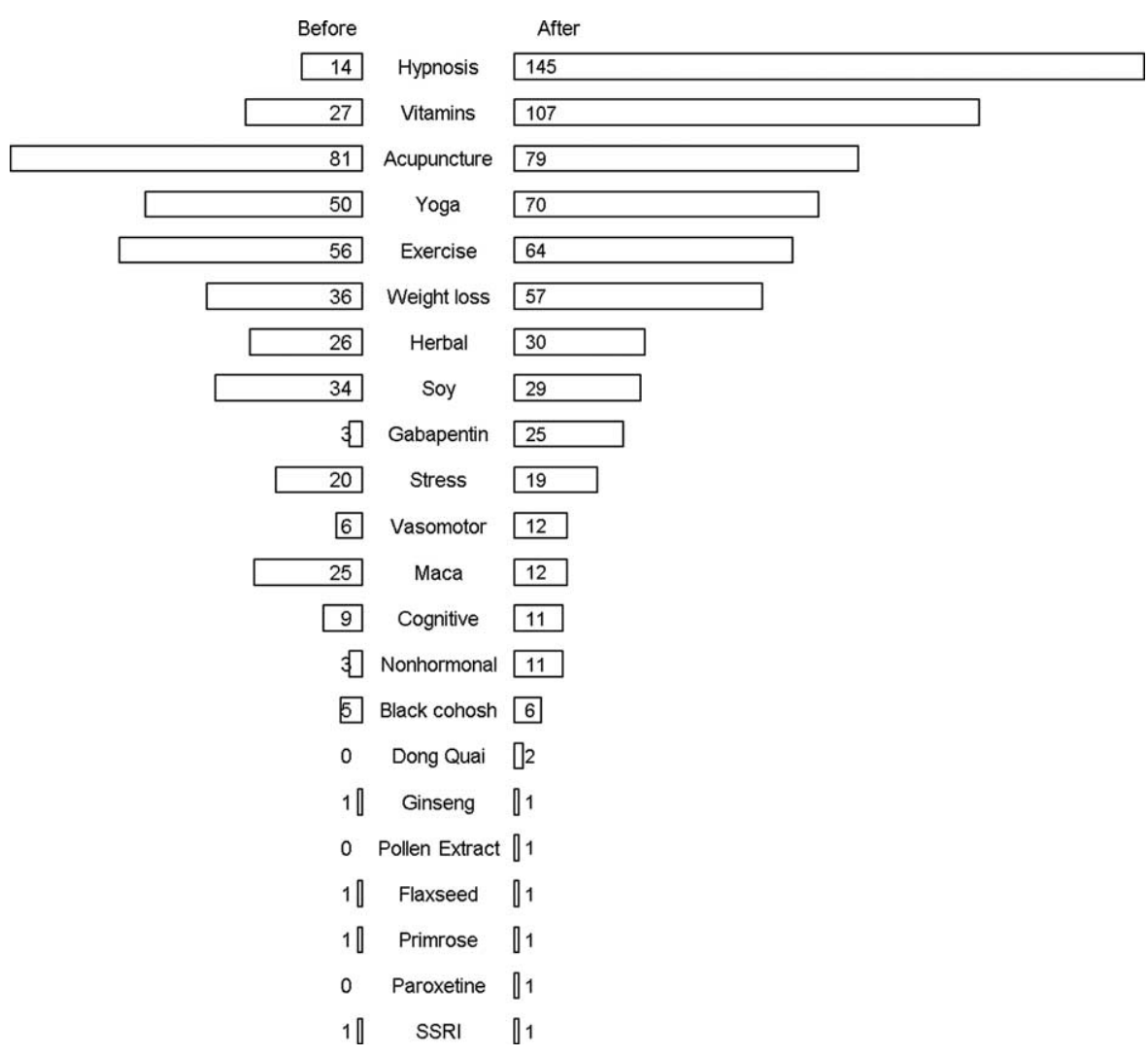

FIG. 2. Keyword frequency on Twitter in the 11 days before and after the North American Menopause Society (NAMS) position statement publication. The NAMS position statement was released on September 23, 2015; before period on the left: September 12, 2015 to September 22, 2015, and after period on the right: September 23, 2015 to October 3, 2015. SSRI, selective serotonin reuptake inhibitor.

were published in foreign news outlets or blogs (Australia, Malaysia, United Kingdom, Isle of Mann).

Our analysis of temporal diffusion showed immediate but unsustained media attention. Most of the coverage was limited to a couple of days after the press release, suggesting there was no real burst of activity after the initial coverage, other than half a dozen articles published during a period spanning from 2 to 5 months thereafter.

In addition, the position statement coincided with a change in the menopause conversation on Twitter with an increase in the top four keywords menopause, hot flashes, NAMS, and hormonal. Tweets pertaining to hypnosis, one of the few nonhormonal therapies recommended by the position statement, increased 10-fold after the position statement was released. Whereas these occurrences are a small fraction of the total universe of tweets, the burst could suggest that the recommendation of that therapy was interesting to the users and that further publication on the topic could be well received. We cannot clearly state that one caused the other, but the temporal pattern of the change in topics is notable.

Our study findings should be interpreted in light of some limitations. Overall, we studied the diffusion of knowledge or transfer of information, which is foundational for, but substantially different from, the actual use of this knowledge in practice. ${ }^{6}$ In our Google searches, we did not include nonEnglish words, which may have limited our ability to find press coverage in non-English-speaking North American regions (eg, Mexico) or internationally. Therefore, we cannot estimate the extent at which the position statement attracted international attention. Whereas the tweets were initially filtered by the top keywords, it is possible that tweets not relevant to the topic could have been included. Also, there may have been external variables influencing the frequency of the tweets' keywords in the two periods. Last, analysis of the geographic dispersion was limited only to NPR coverage; it would be informative in the future to include audience size and distribution from other media outlets as well. We coded the NPR stations using state and census boundary lines, but acknowledge that stations can air across state lines and for some states across census regions. It may also be useful to obtain statistical information on the number of NPR listeners or readers who use Twitter to derive a direct correlation of this source to the Twitter data.

\section{CONCLUSIONS}

The goal of this study was to depict the temporal, geospatial, and topical diffusion of the NAMS position statement and its content. We found broad, national coverage of the 


\section{CARPENTER ET AL}

statement within the United States primarily due to syndicated NPR coverage. We found that the position statement was covered by the media over a short time period, mainly over the days immediately after the statement release. The appearance of the position statement coincided with changes in the menopause conversation on Twitter. Our methods and findings may be useful for directing the development and diffusion of future NAMS position statements and/or press releases.

Acknowledgments: The authors thank Nirmala Xavier, $\mathrm{PhD}$, for editorial assistance.

\section{REFERENCES}

1. North American Menopause Society. Nonhormonal management of menopause-associated vasomotor symptoms: 2015 position statement of The North American Menopause Society. Menopause 2015;22:1154-1174.

2. Rogers E. Diffusion of Innovations. 5th ed. New York: Free Press; 2003.

3. Gephi makes graph handy; 2016. Available at: https://gephi.org. Accessed July 15,2016

4. Sci2 Team. Science of Science (Sci2) Tool. Available at: http://sci2 cns.iu.edu. Accessed July 15, 2016.

5. Federal Communications Commission. FM query broadcast station search Available at: https://www.fcc.gov/media/radio/fm-query. Accessed July $15,2016$.

6. Gonzales R, Handley MA, Ackerman S, O'Sullivan PS. A framework for training health professionals in implementation and dissemination science. Acad Med 2012;87:271-278 